\title{
PENOSCULATING CONICS OF A PLANE CURVE*
}

\author{
BY J. W. LASLEY, JR.
}

1. The Penosculating Parabolas. There is a one-parameter family of parabolas on three non-collinear points of a plane curve. If two of these three points approach a third point at which the tangent to the given curve is well-defined and the curvature is definitely non-vanishing, there is determined a oneparameter family of penosculating parabolas at the point. Abel Transon $\dagger$ found that the directrices of these parabolas all pass through a point on the normal to the curve at the given point $P$ on the convex side of the curve whose distance from $P$ is equal to one-half the radius of curvature. He found that the locus of the foci of these parabolas is a circle whose diameter is that half of the radius of curvature that terminates at $P$. Transon obtained a construction for one of the parabolas, the osculating parabola. We shall now generalize this construction so as to make it applicable to any parabola of the family.

For this purpose we shall associate with each parabola of the pencil a line on $P$, parallel to the axis of the parabola. We shall call this line the axis parallel, and the pencil of lines to which it belongs the axis pencil. To each line of the axis pencil there corresponds a unique parabola of the pencil of penosculating parabolas except in the case of the tangent to the given curve. We shall make correspond to this tangent the degenerate parabola consisting of that tangent counted twice. In this way we have set up a one-to-one correspondence between the parabolas of the pencil and the axis parallels. In this correspondence there corresponds to the normal as an axis parallel a parabola whose axis is that normal, whose tangent at its vertex is the tangent to the given curve, and whose focus is the center of the circular locus of the orthogonal projections of the center of curvature on the axis parallels.

To construct a parabola of the pencil of penosculating parabolas we proceed as follows. Project orthogonally the center of curvature $C$ of the given curve at $P$ upon the axis parallel $a$ cor-

* Presented to the Society, February 28, 1931.

† Journal de Mathématiques, vol. 6 (1841), p. 191. 
responding to this parabola, obtaining the point $D$. Project orthogonally $D$ upon the normal at $P$ into a point $E$. The line on $E$ parallel to $a$ is the axis of the required parabola. The focus of the parabola will lie on a line $s$ through the point $P$ which makes with the normal an angle equal to that made by the axis parallel $a$, but on the opposite side. This line $s$ we shall call the symmetric line for the axis parallel. The focus is then the point of intersection $F$ of the axis of the parabola and the symmetric line. Let $A$ be the point on the normal at $P$ on the convex side of the curve whose distance from $P$ is equal to one-half the radius of curvature. The directrix of the parabola is the line on $A$ perpendicular to the axis of the parabola. The focus and directrix of the penosculating parabola having been located, its construction may now be regarded as complete. Of course, the locus of intersections of the axis parallels and corresponding directrices is a circle tangent to the given curve at $P$. This circle has a diameter equal to one-half the radius of curvature, and is consequently the reflexion in the tangent of the circular locus of foci of penosculating parabolas. If in the foregoing construction for any penosculating parabola the axis parallel is taken to be the axis of aberrancy of Transon, we obtain the osculating parabola. That the construction can be generalized by having the axis parallel play the role of the axis of aberrancy seems not to have been noticed.

2. The Penosculating Eguilateral Hyperbolas. There is also at $P$ a one-parameter family of penosculating equilateral hyperbolas obtained from the pencil of equilateral hyperbolas on three non-collinear points of a given curve by letting two of the three points approach the third. These hyperbolas seem to have received practically no attention. Cesàro* obtained for one of them, the osculating equilateral hyperbola, a canonical form for its equation referred to a local reference frame. We shall extend the correspondence outlined above so as to include these hyperbolas and give a construction available for any penosculating equilateral hyperbola of the pencil.

The locus of the centers of these hyperbolas is a circle on the convex side of the given curve with diameter equal to the radius of curvature. This circle touches the given curve at $P$ and has,

\footnotetext{
* Lezioni di Geometria Intrinseca, Naples, 1896, p. 59.
} 
then, the same normal there. It is the reflexion in the tangent of the circular locus of projections of the center of curvature on the axis parallels. Moreover, the center of this circle is the vertex of the pencil of directrices of penosculating parabolas mentioned by Transon. We shall make use of this circle to extend our correspondence to include the hyperbolas. For this purpose we make correspond to each axis parallel that hyperbola whose center is the further intersection of the axis parallel with the circular locus of centers of penosculating equilateral hyperbolas. To each hyperbola there is a unique axis parallel, namely that determined by the center of the hyperbola and $P$. In this correspondence we shall assign to the tangent as an axis parallel the degenerate hyperbola consisting of the tangent and the normal to the given curve at $P$. To the normal there will correspond a hyperbola whose transverse axis is the normal and whose conjugate axis is parallel to the tangent and distant from it on the convex side of the curve an amount equal to the radius of curvature. For this hyperbola we have a very simple construction for obtaining its asymptotes and axes. With $P$ as a center and with the radius of curvature as radius describe a circle. This circle meets the tangent in points which joined to $P$ give the asymptotes of the hyperbola; it meets the normal in points which joined to $P$ give the axes of the hyperbola. Of course, the above refers only to the hyperbola corresponding to the normal, but it will be seen below that it is a special case of a more general construction available for all penosculating equilateral hyperbolas.

To construct any hyperbola of the pencil we proceed as follows. With $P$ as a center and with a radius equal to the projection of the radius of curvature on the corresponding axis parallel describe a circle. This circle passes through the center of the required hyperbola as picked out by the further intersection of the axis parallel with the locus of centers. This circle meets the tangent to the given curve at $P$ in points which joined to the center give the asymptotes; it meets the normal in points which joined to the center give the axes. Since we know that the required hyperbola goes through $P$, and now have a construction for its asymptotes, we may consider the construction for any penosculating equilateral hyperbola complete. It is interesting to note that for every hyperbola of the pencil it is so that the 
projection of the radius of curvature of the given curve upon the axis parallel is a mean proportional between the intercepts of the tangent of the hyperbola on the tangent. If, in particular, in the foregoing construction, the chosen axis parallel is the axis of aberrancy of Transon, the hyperbola obtained is the osculating equilateral hyperbola of Cesàro. Our correspondence, then, makes correspond the osculating parabola, the axis of aberrancy and the osculating equilateral hyperbola.

3. Transon's Parabola. There is at an ordinary point of a curve a one-parameter family of penosculating conics obtained from the pencil of conics on four points by letting three of the points approach the fourth. Wilczynski* has pointed out that among these conics there will ordinarily be one, and only one, parabola, the osculating parabola. There will also be one, and only one, equilateral hyperbola, the osculating equilateral hyperbola. Wilczynski notes, too, that there will be one, and just one, conic for which the order of contact at the point will rise to four. This conic he calls the osculating conic. Transon found that the locus of centers of penosculating conics is the axis of aberrancy. He found also that the axes of these penosculating conics envelop a parabola, which we shall call, with Wilczynski, Transon's parabola. Due to Transon also is the fact that the focus of this parabola is the orthogonal projection $G$ of the center of curvature of the given curve upon the line joining $P$ to the focus of the osculating parabola, that is, upon the line we have called the symmetric line for the axis of aberrancy. In the terminology we are employing here this focus appears also as the point of intersection of the symmetric line for the axis of aberrancy and the circular locus of feet of perpendiculars let fall from the center of curvature on the axis parallels. It is, too, the reflexion in the normal of the orthogonal projection of the center of curvature upon the axis of aberrancy. Transon found that the directrix of this parabola is the axis of aberrancy. Consequently the axis of the parabola is the line on $G$ perpendicular to the axis of aberrancy. It is of interest, though not noted by Transon, that the focus of the osculating parabola lies on the symmetric line for the axis of aberrancy half way between the given point $P$ and the focus of Transon's parabola. The tangent

* This Bulletin, vol. 22, p. 317. 
to Transon's parabola at the vertex always coincides with the axis of the osculating parabola. The tangent at the vertex of the osculating parabola is always parallel to the axis of Transon's parabola, but coincides with it if, and only if, the given curve is a logarithmic spiral whose tangents cut the radii vectores at an angle whose cotangent is $\pm 3 \sqrt{ } 2$. The directrix of the osculating parabola coincides with the axis of Transon's parabola if, and only if, the given curve is a logarithmic spiral with an angle whose cotangent is $\pm 3 \sqrt{ } 3$. The symmetric line for the axis of aberrancy is usually quite different from the axis of Transon's parabola. It coincides with it if, and only if, the given curve is a logarithmic spiral whose angle has \pm 3 for its cotangent. In this event the aberrancy of the given curve is unity at the point, the vertex of Transon's parabola coincides with the focus of the osculating parabola and Transon's parabola touches the tangent and the normal of the given curve at the ends of the latus rectum. Wilczynski noted that the tangent and the normal of the given curve always touch Transon's parabola. It may be added that the normal always touches it at the center of curvature, and that a line joining the center of curvature to the focus of the osculating parabola always meets the tangent to the given curve in a point $T$ at which Transon's parabola touches that tangent. The reflexion $J$ of the center of curvature $C$ in the tangent when joined to $T$ gives that diameter of Transon's parabola which bisects all chords parallel to the tangent.

4. Construction for a Penosculating Conic. By means of Transon's parabola we can now obtain a construction for any penosculating conic. Wilczynski noted for the osculating conic a property true for all penosculating conics, namely, that the tangents to Transon's parabola from a point on the axis of aberrancy are the axes of the conic which has that point as center. It turns out that one of these axes always touches Transon's parabola between the points at which that parabola touches the tangent and the normal to the given curve. This axis is the axis on which lie the foci of the conic. If now a circle be described upon that segment of the other axis included between its intersections with the tangent and the normal of the given curve, this circle will meet the first named axis in the foci of the desired penosculating conic. Since this conic is known to pass through 
the point $P$ on the given curve, we now know the foci and one point, and the conic can readily be drawn. With the exception of the osculating parabola, this construction applies to any member of the pencil of penosculating conics. Since a construction for the osculating parabola has been outlined above, we may regard the problem of constructing penosculating conics as completely solved.

5. The Ellipse of Minimum Eccentricity. Wilczynski showed that among the conics just considered there is an ellipse of minimum eccentricity. We can now obtain the following construction for this ellipse. Let us project orthogonally the center of curvature of the given curve upon the axis of aberrancy. This projection is the center of the ellipse of minimum eccentricity. With the position of the center known we can construct the ellipse by means of the construction just outlined for penosculating conics in general. It is interesting to note that a circle with center at $P$ and with radius equal to the semi-diameter of this ellipse which terminates at $P$ meets the axis of aberrancy again in the center of the osculating equilateral hyperbola, and meets the symmetric line for the axis of aberrancy on the concave side of the given curve in the focus of Transon's parabola. Penosculating ellipses of equal eccentricity occur in pairs. Wilczynski found that the centers $O_{1}$ and $O_{2}$ of any two of these ellipses of equal eccentricity are so situated on the axis of aberrancy that if $O$ denotes the center of the ellipse of minimum eccentricity, $P O$ is the geometric mean between $P O_{1}$ and $P O_{2}$. That is, the centers of the ellipses of equal eccentricity form an involution whose center is the center of the ellipse of minimum eccentricity. Although for a given suitable value for the eccentricity there are two ellipses that have that eccentricity, this is not true of the penosculating hyperbolas, if we confine our attention to the eccentricities corresponding to the real foci and real directrices. Although an ellipse has only one real eccentricity, the hyperbola ordinarily has two. For a given suitable value for the eccentricity of an hyperbola there are two penosculating hyperbolas, one with this eccentricity for its real foci and real directrices, the other with this eccentricity for its imaginary foci and imaginary directrices. If now we associate in pairs those hyperbolas which have equal eccentricities, we can extend the 
above involution to the entire family of penosculating conics. If, further, we define the cross-ratio of four members of a oneparameter family of conics to be that of the parameters which single them out in the family, we can conclude that the pairs of conics of equal eccentricity separate harmonically the osculating equilateral hyperbola and the ellipse of minimum eccentricity. In this involution of penosculating conics so determined the partner of the osculating parabola is the degenerate parabola consisting of the tangent to the given curve counted twice. Although as Wilczynski found, there is in this family a unique ellipse of minimum eccentricity, there is no hyperbola of maximum eccentricity. The osculating equilateral hyperbola and the ellipse of minimum eccentricity appear as the double conics in this involution of penosculating conics.

The University of North Carolina

\section{THE BITANGENTIAL CURVE*}

BY T. R. HOLLCROFT

1. Introduction. The bitangential curve of an algebraic surface is the locus of the points of contact of planes which touch the surface in two points. It is the reciprocal of the nodal developable. The order of this curve, for an algebraic surface without singularities of any kind, has been determined by Cayley, $\dagger$ who called it the node-couple curve.

When the surface has a nodal and a cuspidal curve, each of given order, the orders of the spinodal and flecnodal curves were found by Cayley. $\ddagger$ The order of the bitangential curve of a surface with nodal and cuspidal curves, however, has not been found explicitly. Basset§ makes the following statement: "I have not succeeded in ascertaining the reduction in the degree of the bitangential curve which is produced by a nodal and a cuspidal curve; but if the reduction is denoted by $x b+y c$, the method of the preceding paragraph indicates that $x$ and $y$ are

* Presented to the Society, December 30, 1930.

$\dagger$ A. Cayley, Collected Mathematical Papers, vol. 6, p. 346.

¥ A. Cayley, loc. cit., vol. 6, pp. 342-343.

$\S$ A. B. Basset, A Treatise on the Geometry of Surfaces, Cambridge, 1910, p. 280. 